\title{
High Aspect Ratio Graphene Nanosheets Cause a Very Low Percolation Threshold for Polymer Nanocomposites
}

\author{
R. JAN*, A. Habib And H.Y. AbBasi \\ School of Chemical and Materials Engineering, National University of Sciences and Technology, \\ H-12 Campus, Islamabad, 44000, Pakistan
}

\begin{abstract}
Liquid exfoliated, high aspect ratio (1272) graphene nanosheets (GNS) are dispersed in thermoplastic polyurethane (TPU) to prepare range of nanocomposites. A three fold increase in direct current conductivity is recorded at 0.0055 volume fraction $\left(V_{\mathrm{f}}\right)$ of GNS-TPU composites as compared to pristine TPU. It is suggested that the percolation threshold for conducting network achieved at low filler loadings is due to the high aspect ratio and homogeneous dispersion of GNS within the polymer. The experimental results are interpreted using interparticle distance model and modified power law. The two models predict threshold filler loading in $0.015-0.001$ range volume fraction GNS based on the average values of mean length and no. of layers per nanosheet. The experimental results favor modified power law as it relies on aspect ratio of fillers. A slight deviation in our study from modified power law may be due to aggregation in as prepared GNS.
\end{abstract}

DOI: 10.12693/APhysPolA.129.478

PACS/topics: 72.80.Tm

\section{Introduction}

Owing to its amazing electrical, thermal and mechanical properties, graphene has been utilized in several polymer matrices for different applications but its role and properties as hybrid material in the polymers has not been completely understood yet $[1,2]$. One such property is its percolation effect as conducting filler in various polymers. The empirical explanations are yet to be correlated with the desirable theoretical approach [3]. Along with the analytical approaches, the experimental work based on graphene as conducting inclusion inside insulating matrices has drawn a wide range of efforts with a considerable success. The various aspects considered for GNS-polymer composites to achieve enhanced electrical characteristics are chemical modifications of GNS to improve the dispersion state and route in polymers, and the structure and nature of the polymers [3, 4]. The percolation effect arises at a specific volume fraction of conducting filler dispersed in a polymeric matrix. At this particular volume fraction, termed as percolation threshold, an electrical network is formed in an otherwise insulating material. A scaling law relationship is applied to the electrical conductivity of the nanocomposites near the percolation threshold [5]:

$$
\begin{aligned}
& \sigma=\sigma_{0}\left(\frac{V_{\mathrm{f}}-V_{\mathrm{c}}}{1-V_{\mathrm{c}}}\right)^{t} \text { for } V_{\mathrm{c}}>V_{\mathrm{f}}, \\
& \sigma=\sigma_{0}\left(\frac{V_{\mathrm{c}}-V_{\mathrm{f}}}{V_{\mathrm{c}}}\right)^{-s} \text { for } V_{\mathrm{c}}<V_{\mathrm{f}},
\end{aligned}
$$

where $\sigma$ is the effective electrical conductivity, $\sigma_{0}$ is the filler intrinsic conductivity, $V_{\mathrm{f}}$ is the filler volume fraction,

*corresponding author; e-mail: rahimjan@scme.nust.edu.pk
$V_{\mathrm{c}}$ is the percolation threshold, $t$ is the 1st percolation exponent and $s$ is the 2 nd percolation exponent. The above Eqs. (1) and (2) do not provide any information about the dimensions of filler, its interaction with the polymer and the dispersion state of filler inside polymer. According to many reports, size and shape of the filler are the driving factors for the determination of percolation threshold. An analytical model, based on the interparticle distance (IPD) of the nanosheets dispersed in the polymer, was developed to predict the percolation threshold $V_{\mathrm{c}}$ for polymer nanocomposites is given below $[6,7]$ :

$$
V_{\mathrm{c}}=\frac{27 \pi D^{2} t}{4(D+\mathrm{IPD})^{3}} .
$$

$D$ is the diameter and $t$ is the thickness of the filler used as conducting reinforcement. Three assumptions considered in the IPD model are (a) the perfect dispersion, (b) the perfect bonding of nanosheets with the polymer matrix, and (c) perfectly similar shape of the nanosheets. In a recent development, Mutlay et al. proposed a phenomenological correlation for the percolation threshold considering the aggregation effects qualitatively [3]:

$$
V_{\mathrm{c}}=\kappa \frac{t}{D}
$$

$\kappa$ determines the degree of aggregation, higher it is, more is the chance of nanosized filler aggregation in the polymer matrix. Equation (4) is a part of modified power law [3] given as

$$
\sigma=\sigma_{\mathrm{GNS}}\left(V_{\mathrm{g}}, T\right)\left(V_{\mathrm{f}}-\kappa\left(\frac{t}{D}\right)\right)^{2-\frac{11}{42}(6-d)+2 \varepsilon\left(\frac{t}{D}\right)}
$$

In the modified power law, the conductivity is measured as a function of filler aspect ratio, spatial distribution in polymer matrix, moderate gate voltage and temperature. Here in our work GNS are homogeneously dispersed in TPU and DC conductivity measurements are used for the evaluation of the percolation threshold of 
nanocomposites with a range of GNS volume fractions at room temperature. All the analytical approaches mentioned above are utilized for possible prediction of percolation threshold. The percolation threshold value of 0.0055 volume fraction of GNS (pristine) may be the lowest value for such polymeric composite system with liquid exfoliated GNS.

\section{Materials and method}

As-supplied graphite powder (Branwell Graphite Limited, grade RFL 99.5) is added to $60 \mathrm{ml}$ of $N$-methylpyrrolidone $(20 \mathrm{mg} / \mathrm{ml})$. This mixture is sonicated for $48 \mathrm{~h}$ using a flat head probe sonic tip. The procedure yields exfoliated GNS of high quality [8]. To remove any unexfoliated graphite and size selection, the dispersion is centrifuged (HettichMikro 22R) at 500, $300 \mathrm{rpm}$ for $45 \mathrm{~min}$ and the supernatant is collected [9-11]. The supernatants from the two centrifugation rates are then filtered through PET membranes (pore size $0.4 \mu \mathrm{m}$ ) and dried overnight at $60^{\circ} \mathrm{C}$. The $300 \mathrm{rpm}$ supernatant powder is used for the formation of composites after dispersing it in tetrahydrofurane (THF) by mild bath sonication $(2 \mathrm{mg} / \mathrm{ml})$. To assess the exfoliation state of the dispersed GNS and to measure its dimensions, a few drops of dispersion are dropped onto holey carbon grids (400 mesh) and analyzed using a JEOL 2100 TEM at $200 \mathrm{kV}$. To make composites films, TPU (BASF) is dissolved in THF $(100 \mathrm{mg} / \mathrm{ml})$ by overnight stirring and various volumes of the GNS stock dispersion are added to TPU solution. Each dispersion is sonicated for $4 \mathrm{~h}$ in sonic bath (Branson 1510E-MT sonic bath), followed by drop casting into Teflon trays. In all cases, the total liquid volume and solids mass are kept constant $(160 \mathrm{mg})$ to avoid any drying related variation. The composites are dried at $25^{\circ} \mathrm{C}(24 \mathrm{~h})$ followed by another drying period at $65^{\circ} \mathrm{C}(72 \mathrm{~h})$.

\section{Results and discussion}

Liquid exfoliation process is a proficient way of obtaining dispersions with a large quantity of defect free GNS [8, 12]. By performing few processing steps on these dispersions make them ideal for the formation of composites. The electrical characteristics of composites are vigorously driven by the size of the filler particles, in particular the aspect ratio $[3,13,14]$. We have been able to get large aspect ratio GNS by utilizing centrifugation technique [12]. To analyze the size of the GNS quantitatively, transmission electron microscope (TEM) is used. As evident from TEM image in Fig. 1a, we obtain few layers GNS by estimating the number of layers per nanosheet, $N$. The data for length distribution of $>150$ nanosheets is presented as histogram in Fig. 1b showing that the mean nanosheet length is approximately equal to $1.4 \mu \mathrm{m}$. The approximate analysis done with the TEM shows GNS to be 1-10 monolayers thick having mean values around $N \approx 3$.
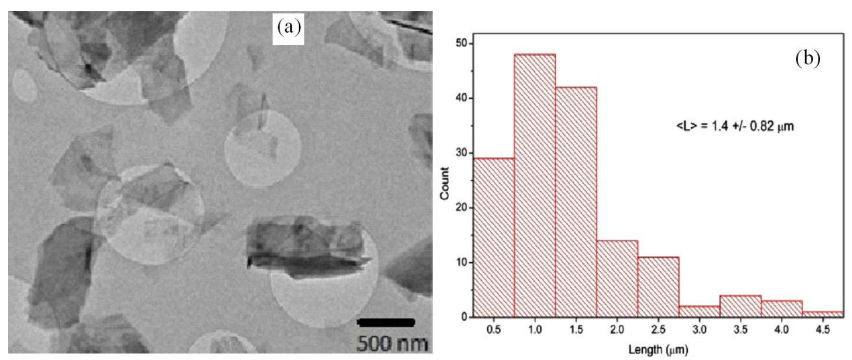

Fig. 1. Transmission electron microscopy characterization of exfoliated, size-selected GNS: (a) representative TEM image (b) histogram found by measuring the lengths of $>150$ nanosheets from the TEM images.
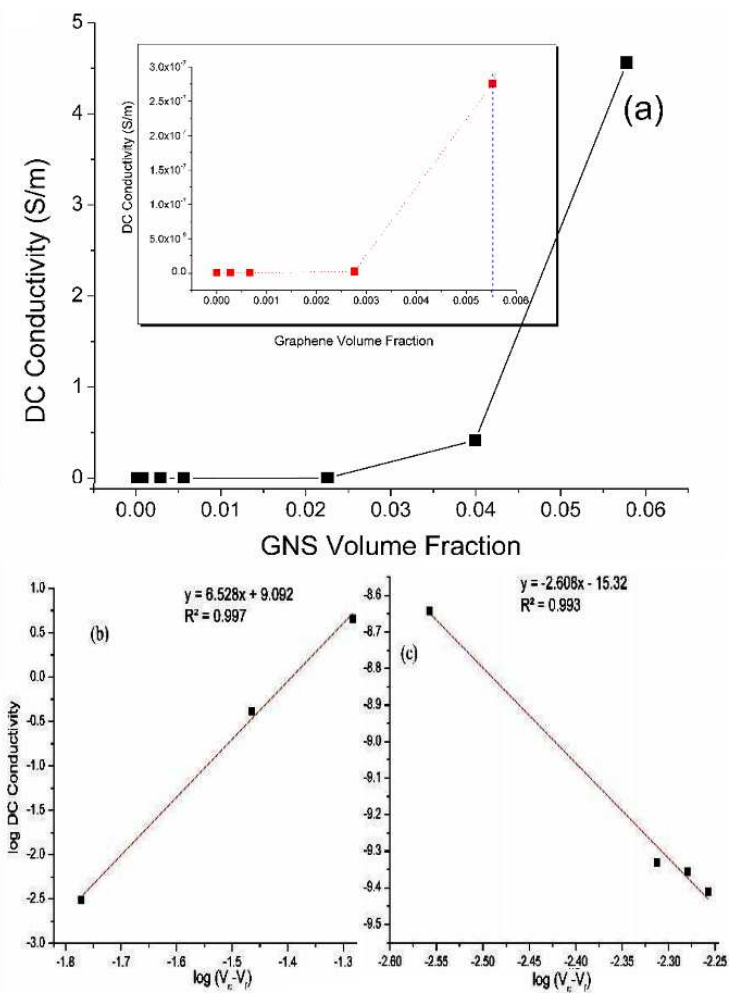

Fig. 2. (a) DC conductivity behavior of TPU-GNS nanocomposite as a function of GNS volume fraction. The overall volume range is up to $0.06 V_{\mathrm{f}}$ GNS with inset showing 3 -fold increase in electrical conductivity at $0.0055 V_{\mathrm{f}}$ GNS. The effect is studied at room temperature. $(\mathrm{b}-\mathrm{c})$ The percolation threshold data fitting for conductivity as a function of volume fraction (b) above the percolation threshold $\left(V_{\mathrm{c}}\right)$ and $(\mathrm{c})$ below the percolation threshold $\left(V_{\mathrm{c}}\right)$.

The DC electrical conductivity measurements are performed on Keithley-6487 source meter with voltage range $0-3 \mathrm{~V}$. The nanocomposite films are cut in $2.25 \mathrm{~mm} \times$ $30 \mathrm{~mm}$ rectangular shapes. Figure 2 a clearly shows that by adding only a very small amount of GNS, conductivity is on the increase. At lower GNS loadings, the electrical conductivity remains very close to the pure polymer conductivity value. However, at $0.0055 V_{\mathrm{f}}$ GNS and after this point the electrical conductivity increases as a 
function of graphene volume fraction. We have analyzed the data in terms of Eqs. (2) and (3) to find the values of the percolation exponents $t$ and $s$, the data fits well over the entire range for the GNS-TPU composites. The values for both the percolation exponents are $t=6.53$ and $s=2.61$ evident from Fig. 2b,c. The values of both $t$ and $s$ are considerably high as compared to the standard values for both $2 \mathrm{D}$ and $3 \mathrm{D}$ nanocomposites which lies in the range $1-2$. The reason for this deviation may be the electron hopping to the adjacent sites which is the main factor responsible for the rapid increase in the electrical conductivity of nanocomposite. This effect takes place when the IPD is equal to or less than $10 \mathrm{~nm}[6]$.
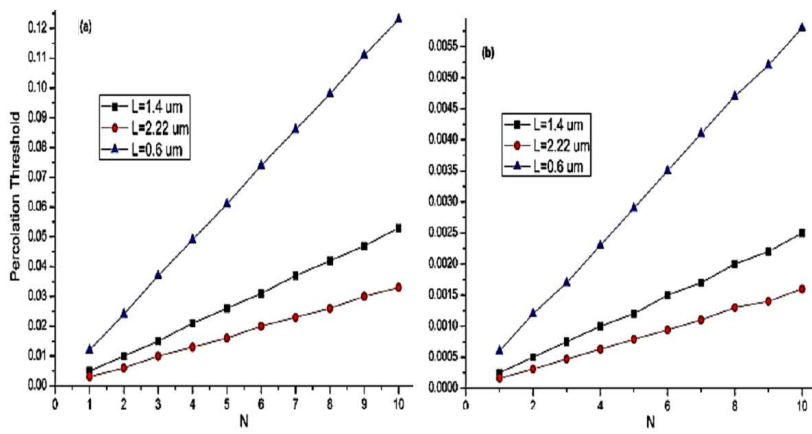

Fig. 3. The trend lines for the experimental percolation threshold data based on (a) the IPD model and (b) a part of the modified power (assuming $\kappa \approx 1$ ) law as a function of number of layers per flake $(N)$ for various mean lengths of the GNS.

Figure 3a,b presents a full range of the percolation threshold values for our results based on the IPD model and a part of modified power law. The empirical data is attained as a function of number of layers per nanosheet, $N(1-10)$ for the mean nanosheet length $L(1.4 \mu \mathrm{m})$, along with its lower and upper extremes $( \pm 0.82 \mu \mathrm{m})$. The theoretical value for monolayer graphene sheet thickness is found to be around $0.035 \mathrm{~nm}$ [15]. Considering the IPD model to predict the percolation threshold $\left(V_{\mathrm{c}}\right)$ using Eq. (3), the best fit value close to the experimental value is found to be $0.006 V_{\mathrm{f}}$ GNS for the case when the $L$ value is considered to be at the maximum limit $(2.2 \mu \mathrm{m})$ and $N \approx 2$. At this particular instance we can state that all the assumed criterion of the IPD model have been fulfilled in our case. Based on the synthesis routes for both GNS and then nanocomposites, we can claim homogeneous dispersion and good bonding but TEM analyses give a varied flakes length and a range of GNS layers. Thus we consider the nanosheets mean length $(L \approx 1.4 \mu \mathrm{m})$ and the average number of layers value $(N \approx 3)$; the percolation threshold $\left(V_{\mathrm{c}}\right)$ value comes out to be $\approx 0.015$. This value is a bit farther than the experimentally found value. For monolayer and twolayer GNS, the percolation threshold value lowers down to 0.005 and $0.010 V_{\mathrm{f}}$ of GNS as shown in Fig. 3a. Modified power law (Eq. (5)) is also based on the aspect ratio of the filler inside the polymer matrix and the experimen- tal results can be explained by using a part of it (Eq. (4)). It can be distinguished from the IPD model by the added facet of the aggregation factor $\kappa$. Considering the average mean length value of GNS $(1.4 \mu \mathrm{m})$ and no. of nanosheets per flake $(N) \approx 3 \div 4, V_{\mathrm{c}} \approx 0.0055$, we get the aggregation factor value $6.87-9.16$. It has been predicted though that for few layers GNS, $\kappa$ is $\leq 1$. If we assume the value of aggregation factor to be around 1 , the predicted percolation threshold is $\approx 0.001 V_{\mathrm{f}}$ GNS for $N$ range of $3-4$ as shown in Fig. 3b. The aggregation of the GNS inside the nanocomposite is a key factor due to which the predicted values of percolation threshold are lower than the experimental value. The experimental value for percolation threshold $\left(0.0055 V_{\mathrm{f}}\right.$ GNS) lies in the range predicted by both the models mentioned above but more closer to the modified power law. By considering both the approaches to be in the same category as both rely heavily on the aspect ratio, our experimental findings can be very useful in understanding the two models in the perspective of aggregation of nanofillers occurring inside the polymeric nanocomposites. This work is an important step forward in the field of conducting nanocomposites as we can control the aspect ratio of GNS by using liquid exfoliation technique.

\section{Conclusion}

GNS-TPU nanocomposites are prepared via liquid exfoliation and solution based method to achieve improved dispersions. The high aspect ratio GNS caused a very low percolation threshold of 0.0055 volume fraction graphene in TPU. To the best of our knowledge this is the lowest value ever achieved by using as prepared GNS (liquid exfoliated) without any further post treatment. The results are interpreted via well established scaling law and verified from IPD model and modified power law. The high aspect ratio and layered structure of GNS are the main features along with its homogeneous dispersion in TPU. The results will form a base for some more fruitful work in the graphene-polymer nanocomposites for the applications involving conducting behavior.

\section{References}

[1] S.H. Xie, Y.Y. Liu, J.Y. Li, Appl. Phys. Lett. 92, 243121 (2008).

[2] P.N. Nirmalraj, T. Lutz, S. Kumar, G.S. Duesberg, J.J. Boland, Nano Lett. 11, 16 (2011).

[3] I. Mutlay, L.B. Tudoran, Fullerenes Nanotubes Carbon Nanostruct. 22, 413 (2014).

[4] S. Rana, J.W. Cho, L.P. Tan, RSC Adv. 3, 13796 (2013).

[5] G. Cunningham, M. Lotya, N. McEvoy, G.S. Duesberg, P.V.D. Schoot, J.N. Coleman, Nanoscale 20, 6260 (2012).

[6] J. Li, J.K. Kim, Compos. Sci. Technol. 67, 2114 (2007).

[7] B. Zhang, Y. Yu, Y. Liu, Z.D. Huang, Y.B. He, J.K. Kim, Nanoscale 5, 2100 (2013). 
[8] J.N. Coleman, M. Lotya, A. O'Neill, S.D. Bergin, P.J. King, U. Khan, K. Young, A. Gaucher, S. De, R.J. Smith, I.V. Shvets, S.K. Arora, G. Stanton, H.Y. Kim, K. Lee, G.T. Kim, G.S. Duesberg, T. Hallam, J.J. Boland, J.J. Wang, J.F. Donegan, J.C. Grunlan, G. Moriarty, A. Shmeliov, R.J. Nicholls, J.M. Perkins, E.M. Grieveson, K. Theuwissen, D.W. McComb, P.D. Nellist, V. Nicolosi, Science 331, 568 (2011).

[9] U. Khan, A. O'Neill, H. Porwal, P. May, K. Nawaz, J.N. Coleman, Carbon 50, 470 (2012).

[10] M. Lotya, P.J. King, U. Khan, S. De, J.N. Coleman, ACS Nano 4, 315 (2010).
[11] A. O'Neill, U. Khan, J.N. Coleman, Chem. Mater. 24, 2414 (2012).

[12] P. May, U. Khan, J.M. Hughes, J.N. Coleman, J. Phys. Chem. C 116, 11393 (2012).

[13] P. May, U. Khan, A. O'Neill, J.N. Coleman, J. Mater. Chem. 22, 1278 (2012)

[14] G.E. Padawer, N. Beecher, Polym. Eng. Sci. 10, 18 (1970).

[15] C.C. Chen, W. Bao, J. Theiss, C. Dames, C.N. Lau, S.B. Cronin, Nano Lett. 9, 4172 (2009). 